\title{
TRANSMISIÓN DE INFORMACIÓN POLÍTICA Y ESTRUCTURA DE REDES SOCIALES Un análisis comparativo entre estudiantes universitarios de España y Portugal
}

\begin{abstract}
Resumen: La investigación desarrollada trata sobre la estructura de comunidades virtuales (redes sociales en particular) en estudiantes universitarios. Se pretende avanzar en el modo de agrupación de personas y tránsito de información política en las redes sociales en torno a estructuras de tipo elitista o pluralista, usando la teoría de estructura de comunidades en relaciones públicas de Hunter y Dahl.

Dicha investigación se hace en términos comparativos entre España y Portugal. Resulta especialmente interesante la elección de las Universidades Loyola Andalucía (España) y Algarve (Portugal), ya que se encuentran geográficamente próximas, pero en países distintos y sus jóvenes universitarios presentan características, hábitos y comportamientos propios.
\end{abstract}

Palabras clave: redes sociales, elitismo, pluralismo, Facebook, WhatsApp, Twitter.

\section{DIFFUSION OF POLITICAL INFORMATION AND STRUCTURE IN SOCIAL NETWORKS}

A comparative analysis between spanish and portuguese university students

\begin{abstract}
The research deals with the structure of virtual communities (specially social networks) on university students. The main objective is advancing in how the political information runs in social networks in order to determine elitist or pluralistic structures, using for that purpose the theory of community structures in public relations of Hunter and Dahl.

This research is done in comparative terms between Spain and Portugal. The choice of the Universities Loyola Andalucía (Spain) and Algarve (Portugal), since they are geographically close, but in different countries and their university students have their own characteristics, habits and behaviors.
\end{abstract}

Keywords: Social Network, elitism, pluralism, Facebook,WhatsApp, Twitter.

\footnotetext{
${ }^{1}$ Profesor Adjunto de la Universidad Loyola Andalucía (Espanha)

Doctor en Ciencias de la Información por la Universidad Complutense de Madrid (ajtapia @uloyola.es)

${ }^{2}$ Profesor Adjunto de la Universidad de Algarve (Portugal)

Doctor en Educación Artística/Artes Visuales por la Universidad de Sevilla (fgil@ualg.pt)
} 


\section{Introducción}

Este estudio tiene como antecedente otro con la misma temática, titulado "Los estudiantes universitarios ante las redes sociales: cuestiones de uso y agrupación en estructuras elitistas o pluralistas", publicado en 2010 (Tapia et al., 2010). No obstante, las conclusiones de tal estudio se referían en general a todas las redes sociales, no concretando los resultados a redes sociales específicas como Facebook, WhatsApp o Twitter.

Sin duda, el nivel de desarrollo de las redes sociales entonces (Facebook no se tradujo al español hasta 2008, y WhatsApp fue fundada en 2009 - y comprada por Facebook en 2014) no permitió hacer la distinción entre redes sociales que este estudio propone.

Por ello, como primera gran aproximación, debe señalarse que en esta investigación tratamos de determinar la estructura de las comunidades creadas en redes sociales recuperando la teoría de estructura de comunidades divulgada en los años 50 y 60 por Dahl y Hunter. Una vez analizada la estructura, estudiamos la forma en que transita la información en estas comunidades, destacando el papel de los líderes, los anti-líderes y otras figuras en la generación, distribución y consumo de información en este entorno.

Actualmente, las redes sociales son una fuente preferente de información para los estudiantes universitarios, que las usan como sistema de relación interpersonal y también como fuente de información y demanda de peticiones sociales.

De ellas, Facebook, WhatsApp y Twitter son las redes sociales de mayor uso en España. Sin ir más lejos, según Internet WorldStats (2017) en España hay 23 millones de cuentas de Facebook activas (hay 40 millones de usuarios de internet), y casi 6 millones en Portugal (con 7 millones y medio de usuarios de internet). WhatsApp es usado por 1.200 millones de personas en el mundo, siendo líder en España, donde es usada por el 70\% de la población (Rey, 2017). Twitter por su parte tiene más de 328 millones de usuarios en el mundo(1).

Además, según el estudio anual de redes sociales de IAB Spain (2017), Facebook y WhatsApp son las dos redes sociales preferidas por los españoles. Dicho estudio afianza el total conocimiento de Facebook (100\% de conocimiento sugerido) entre los usuarios de redes so- ciales, seguida por WhatsApp (94\%) y Twitter (92\%).

El uso esencial de WhatsApp y Facebook es social, presentando el perfil de uso de Twitter un uso más informativo. (IAB Spain, 2017).

Otro dato que apoya la importancia de las redes sociales como instrumento de comunicación fundamental entre españoles es que según el mismo estudio el $81 \%$ de los internautas de 16-55 años utilizan redes sociales, lo que representa más de 15 millones usuarios (IAB Spain, 2017).

Así, dado que estamos ante un sistema de comunicación preferente entre la población en general y también entre estudiantes universitarios, consideramos que vehicular la investigación en torno a las redes sociales ofrece unos potenciales resultados aptos que reflejan el modo de convivencia digital en esta población, y nos ayudará a entender cómo se difunde información de tipo político en este ámbito.

Por otra parte, se decidió usar la teoría de estructuras de comunidades de Hunter (1953), que afirmaba que las estructuras preeminentes en comunidades físicas eran de tipo elitista, concebidas fundamentalmente en relación a aspectos como la clase social o la reputación, con presencia muy marcada de la figura del líder, y Dahl (1961), partidario de estructuras pluralistas, más descentralizadas (Grunig y Hunt, 2003: 404-410) dada la extraordinaria trascendencia que dichas teorías han tenido para la investigación de estructuras comunitarias desde los años 20 para politólogos, sociólogos y comunicólogos.

También tenemos en cuenta el amplio estudio de Walton (1966) sobre centralización en comunidades, en la que examinó 39 estudios en 75 comunidades, y llegó a la conclusión de que los sociólogos habían encontrado con más frecuencias estructuras elitistas, y los politólogos estructuras pluralistas. Después examinó más casos (alcanzando a 166) sobre investigadores ajenos a ambas tendencias, y concluyó que el grado de centralización de poder en una comunidad dependía de las características estructurales como el tamaño de la población, la presencia de una reforma gubernamental o la ausencia de propiedad de las grandes empresas de negocios, por lo que ambos (elitistas y pluralistas) tenían razón.

Actualmente no existe un consenso sobre esta cues- 
tión, pero parece que al menos la mayoría de ellos aceptan la teoría de la contingencia de poder de la comunidad, que señala que algunas de ellas encajan en el modelo centralizado (comunidades elitistas) y otras en el descentralizado (comunidades plurales), dependiendo de sus características.

La cuestión no es menor, pues de la estructura que tenga la comunidad dependerá la forma de hacer cualquier acto comunicativo, también político, en ella.

Como antecedente al estudio de estructuras en redes sociales, hay que nombrar el trabajo de Flórez (2008) que destaca varias tipologías de usuarios en función del uso que hacen de las redes sociales; así, señala que los alpha users son aquellos que actúan como catalizadores de opinión y noticias. Serían una o varias personas que propagan información, resultando por ello de extremo interés para la acción política. Asumirían por tanto la posición de liderazgo dentro del grupo. Otro tipo de usuarios serían los bridge users, que actúan de puente entre grupos. En este caso se trata de individuos que cuentan con relaciones potentes en diferentes grupos, lo que hace que puedan ser buenos comunicadores de elementos innovadores. Finalmente, en contraposición de los alpha users, nos encontramos con los omega users, que son individuos donde finalizan los procesos comunicativos, por simple inactividad, rechazo o miedo al cambio. Son por ellos consumidores, no difundiendo ni propagando información en sentido alguno.

También hay que nombrar como antecedente el estudio de Tapia et al. (2010) que concluyó la presencia de estructuras pluralistas con algunos elementos propios de estructuras elitistas, como la presencia de grupos con mayor uso que otros, pero sin la condición de liderazgo, esencial para concluir una estructura de este tipo.

$\mathrm{Al}$ margen de la cuestión de la estructura de grupos en redes sociales, hay que señalar que abundan los estudios sobre comunicación política y redes sociales. En relación a Facebook, destaca un estudio que concluyó un escaso uso de esta herramienta por parte de los diputados españoles (Túñez y Sixto, 2011).

La red social Twitter ha sido de igual modo objeto de numerosos estudios. Deltell (2011) hizo un estudio de caso sobre el grupo político Equo con unos resultados desesperanzadores: a pesar de ser la formación política la más seguida y preferida, no consiguió generar voto, cosechando unos pobres resultados electorales.

No obstante, Zarrella, (2010) destacó la tendencia contraria. En efecto, tal estudio concluyó que en el $74 \%$ de las contiendas electorales de Estados Unidos los candidatos con más seguidores alcanzaron mejores posiciones finales.

Y en esta misma línea y posicionamiento con respecto a que la presencia política en redes sociales mejora los resultados del grupo, una investigación realizada sobre las elecciones alemanas de 2009 probaron la correlación significativa y positiva entre en número de tweets que mencionan un partido político y sus resultados electorales (Tumasjan et al., 2010)

Así las cosas, otro autor destaca que si bien el valor de las redes sociales como herramienta predictiva en resultados electorales es indiscutible, la reflexión final que podríamos realizar es un conglomerado de múltiples factores como la imagen, la reputación, la credibilidad o la presencia física del candidato. (Congosto, 2011). En ese sentido, tanto Caldevilla (2009) como Rodríguez y Ureña (2010) destacan que las redes sociales deben ser parte del mix de comunicación en campaña electoral. De hecho hay quien señala que esto ya sucede (Castells, 2008; Cárdenas-Neira, 2014).

Otro estudio sobre una herramienta de social media diferente, YouTube, trató la figura del prosumidor de contenido político en tal red, destacando un perfil que consume mucha información, pero que en todo caso resulta pasivo en generación y distribución de la misma (Berrocal et al., 2014).

En definitiva, esta investigación persigue replicar estos modelos de estructura de comunidades en las redes sociales estudiadas (Facebook, WhatsApp y Twitter) para considerar las mejores estrategias de comunicación políticas en este medio, ya que como se aludía anteriormente la estructura define en buena medida el modo de acercamiento.

\section{Metodología}

El diseño de investigación utilizado es no experimental, exploratorio y descriptivo. Por ello, el proceso ha 
consistido en el examen de paradigmas teóricos en relación al objeto de estudio, aplicando posteriormente el uso de la técnica de encuesta.

La encuesta es escrita y autoadministrada por lo que la muestra la completó de forma autónoma, habiendo un técnico responsable de estudio presente en el momento de la realización.

En dicha encuesta se utilizan preguntas dicotómicas, abiertas, cerradas y basadas en escalas. Se decidió el uso de escalas impares, de tipo acuerdo - desacuerdo en cinco posiciones, posibilitando la neutralidad en la opinión, ya que al ser un estudio a priori interesante para los encuestados se esperó una adecuada respuesta.

Específicamente para cada red social estudiada (Facebook, WhatsApp y Twitter), se usó una escala de tipo acuerdo-desacuerdo de Likert de 5 posiciones que medía la actuación del encuestado en las redes sociales y la percepción que tenía de los demás de su grupo al respecto, ya que el estudio de las estructuras elitistas y pluralistas pivota en torno al concepto de líderazgo. Las escalas están basadas en dos investigaciones anteriores: una que trataba las redes sociales de forma genérica (Tapia et al., 2010) y otra que estudiaba Facebook (Ellison, Steinfield y Lampe, 2007).

Por ello, consideramos que condición necesaria para que exista dicho liderazgo en redes sociales es que éste debe ser explícitamente reconocido como tal por el resto, o al menos debe reconocerse por los demás que alguien orienta al resto, no siendo suficiente que alguien use más algún aspecto de dicha red social para ser reconocido como líder.

Es interesante distinguir si en efecto es una sola persona o un grupo de personas el que usa más la red social y orienta el devenir de las conversaciones, ya que de ser de un grupo de personas el que dirige la susodicha podría inferirse la hipótesis del pluralismo como estructura preeminente, mientras que si dicha red social está dominada por una única persona que es reconocida como líder (que a su vez designará un equipo de personas como acólitos), habría que destacar la hipótesis contraria y situar el elitismo como estructura preferente.

En concreto los ítems que se usaron por red fueron los siguientes:

\section{Facebook}

- Soy el que más uso Facebook de mis amigos

- Uno de mis amigos de Facebook sube y comenta más fotos y noticias que los demás

- Uno de mis amigos de Facebook sube y comenta más fotos y noticias políticas que los demás

- Uno de mis amigos de Facebook envía más mensajes de messenger que los demás

- En Facebook tengo varios amigos que comparten bastante más noticias y vídeos que los demás

- En Facebook tengo varios amigos que comparten bastante más noticias y vídeos políticos que los demás

- Uno de mis amigos usa en general más Facebook que los demás

- El que orienta las conversaciones fuera de la red también la orienta en Facebook

-En Facebook nadie orienta las conversaciones

\section{WhatsApp}

- Hay alguien en mis grupos de WhatsApp que sube y comenta más fotos que los demás

- Hay alguien en mis grupos de WhatsApp que sube y comenta más fotos políticas que los demás

- Hay alguien en mis grupos de mis grupos de WhatsApp que publica y comenta más noticias que los demás

- Hay alguien en mis grupos de mis grupos de WhatsApp que publica y comenta más noticias políticas que los demás

- Hay alguien que en general usa más WhatsApp que los demás

- Soy el que más creo grupos y uso WhatsApp de mis amigos

- Creo grupos y uso WhatsApp con más intensidad que la mayoría de mis amigos

- Mis amigos y yo usamos WhatsApp con intensidad parecida

- El que orienta las conversaciones fuera de la red también la orienta en WhatsApp

- Hay alguien en mis grupos de WhatsApp que orienta las conversaciones 


\section{Twitter}

- Uno de mis amigos sube, comenta y retuitea más noticias y fotos que los demás en Twitter

- Uno de mis amigos sube, comenta y retuitea más noticias y fotos políticas que los demás en Twitter

- Varios amigos míos suben, comentar y retuitean más información que los demás

- Soy el que más uso Twitter de mis amigos

- Varios amigos míos suben, comentar y retuitean más información política que los demás

- Soy el que más uso Twitter de mis amigos

- Tengo más seguidores que la mayoría de mis amigos

- Mis amigos y yo usamos Twitter con intensidad parecida

- Muchos amigos míos me siguen

- Consumo información política en Twitter en mayor medida que mis amigos

- Retuiteo información política con mayor frecuencia que mis amigos

- Cuando me llega información política, la leo pero no la retuiteo

La elección de las tres redes que se estudian (Facebook, WhatsApp y Twitter) responde a criterios de orden estratégico, ya que apriori las tres son muy distintas: Facebook está muy orientada al entretenimiento, WhatsApp a la interacción entre personas y Twitter hacia al consumo de la información de actualidad.

La muestra que compone la investigación está compuesta por 291 estudiantes universitarios mayores de 18 años de Faro (Portugal), Sevilla y Córdoba (España). El grado de error en las estimaciones se situó en el 6\% con un grado de confianza del 95,5\%.

En concreto está estructurada del siguiente modo:

\section{(Tabla 1)}

En cuanto a la técnica de muestreo, se utiliza la muestra de población por cuotas. Se trataba de asegurar la adecuada representatividad del universo, esto es, se pretende reproducir la estructura de la población a estudiar . El muestreo por cuotas constituye una síntesis de diferentes tipos y como intencionado, en todo caso ha de considerarse no probabilística, por lo que en principio

\begin{tabular}{|c|c|c|}
\cline { 2 - 3 } \multicolumn{1}{c|}{} & Frecuencia & Porcentaje \\
\hline Hombre & 100 & 34,4 \\
\hline Mujer & 191 & 65,6 \\
\hline
\end{tabular}

\begin{tabular}{|c|c|c|}
\hline España & 152 & 52,2 \\
\hline Portugal & 139 & 47,8 \\
\hline
\end{tabular}

Tabla 1 - Muestra de la investigación (Fuente: elaboración propia)

no soporta la generalización de lo investigado. De lo que se deduce que las conclusiones han de tomarse con la debida cautela.

Los resultados se explotaron con soporte informático de análisis estadístico, de acuerdo con un plan de análisis de diversa naturaleza.

\section{Resultados}

El primer hallazgo pone de relevancia una interesante diferencia entre países, destacándose un mayor uso de WhatsApp y Twitter en España, y menor de Facebook. Los datos de consumo de cada red en concreto son los siguientes:

\begin{tabular}{|c|c|c|}
\hline \multirow{2}{*}{ Facebook } & España & 10,26 \\
\cline { 2 - 3 } & Portugal & 14,77 \\
\hline \multirow{2}{*}{ WhatsApp } & España & 17 \\
\cline { 2 - 3 } & Portugal & 7,77 \\
\hline \multirow{2}{*}{ Twitter } & España & 12,80 \\
\cline { 2 - 3 } & Portugal & 8,96 \\
\hline
\end{tabular}

Tabla 2 - Horas /semana por red social (Fuente: elaboración propia)

Además, el uso que se da a las redes es diferente, tal y como pudo sospecharse. Así se aprecia para Facebook un uso más generalista enfocado hacia lo lúdico. Twitter tiene un perfil más basado en la actualidad y lo político, 


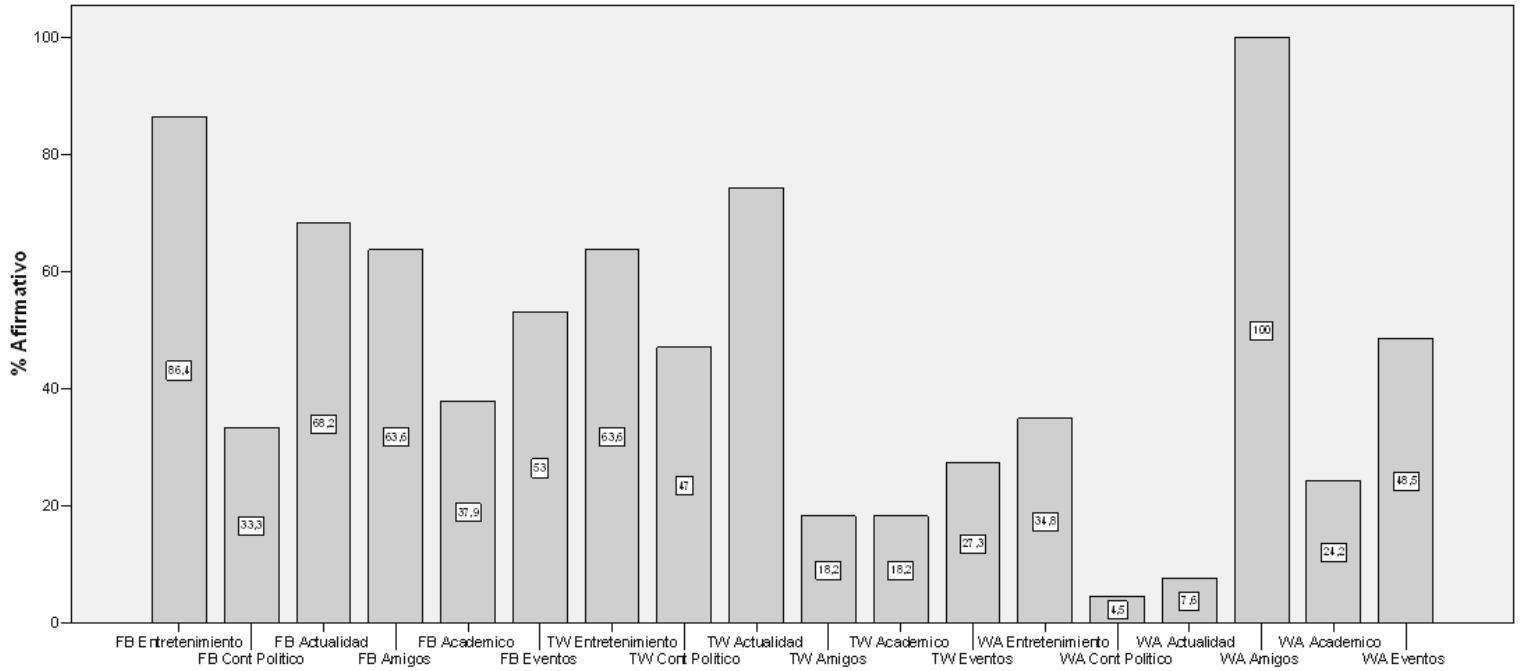

Gráfico 1 - Porcentaje de uso de cada red por elemento (Fuente: elaboración propia)

y finalmente WhatsApp está muy volcado hacia la conversación con amigos.

Los resultados en concreto pueden verse en el gráfico (Gráfico 1).

En relación al ajuste de las mencionadas redes a los modelos pluralistas y elitistas propugnados por Hunter y Dahl, los resultados ponen de manifiesto estructuras diferentes según cada red social estudiada, pero no en relación al país del estudiante.

Así, Facebook se postula como la red más igualitaria de todas. En esta red no se aprecia liderazgo visible (lo que excluye la posibilidad de determinar estructuras elitistas o pluralistas puras), aunque sí perfiles más activos.

En definitiva, la conclusión general gira en torno a la idea de considerar que se dan estructuras pseudopluralistas que incorpora elementos propios de estructuras elitistas como la existencia de subgrupos y también de perfiles individuales especialmente activos, a los que por otra parte no se les otorga la condición de líderes al no ser así reconocidos por el resto.

La red WhatsApp presenta unos resultados que difieren notablemente de los vistos para Facebook. Así, en el caso de WhatsApp se apreciaron estructuras elitistas. En concreto, en más de la mitad de los casos se admite la figura de un líder que orienta las conversaciones, y que además incluye las conversaciones de tipo político.

Finalmente, la red Twitter se manifiesta como la de menor relevancia de las tres estudiadas, tanto en el caso de los estudiantes españoles como portugueses. Se debe entender que Twitter una red de naturaleza dependiente (recordemos en este punto que los ítems de relación son "seguir" o "ser seguido", e implican dependencia en sí mismos), a diferencia de Facebook y WhatsApp (donde la relación entre usuarios es de "amigos" 0 "participantes" de un grupo, lo que denota igualdad), por lo que si en Twitter red se admite seguir con gran intensidad a una persona o varias se debe entender liderazgo de esa persona. En este caso, también se observaron estructuras jerárquicas, más tendente a formaciones elitistas en el caso de España y pluralistas en el caso portugués.

\section{Conclusiones y consideraciones finales}

Los resultados ponen de manifiesto un consumo diferente de las redes al considerar el país, otorgando los portugueses mayor preponderancia a Facebook. Los españoles, en cambio, usan WhatsApp en mayor medida. Además, y dados los resultados específicos para el sistema de mensajería instantánea Messenger, integrado en Facebook, cabe inferir que los estudiantes portugueses usan específicamente el sistema de mensajería instantánea Messenger de Facebook como sustituto de WhatsApp. 
De igual modo, se puso de manifiesto que el uso al que se destinaba cada red es esencialmente distinto, lo que hace considerar el uso complementario de las redes estudiadas en un mix que incluye todas ellas.

Respecto a las estructuras elitistas y pluralistas observadas, hay que destacar en este caso que se perciben estructuras muy distintas según la red social estudiada, pero no según el país de los estudiantes.

Ampliando el radio de acción de la cuestión que nos ocupa, el proyecto tiene sin duda una interesante aplicación a nivel técnico para las redes sociales, ya que conocer la estructura y la forma de difusión de información política determinará tipos de redes sociales y qué técnicas de relaciones públicas son más aptas para vehicular información específica, de una u otra índole a los estudiantes universitarios.

Los beneficiarios fundamentales del proyecto son las organizaciones privadas como públicas: en el caso de las privadas, podrán implementar técnicas de propaganda e información más eficientes en redes sociales; con respecto a las instituciones públicas, pues podrán reali- zar campañas de información y sensibilización mejores en redes sociales. Por otro lado, se hace extensiva también la conclusión a los ciudadanos de cualquier tipo, ya que a través de las campañas recibirán la información de una manera más concreta y adaptada a sus necesidades o usos.

Futuras investigaciones complementarias podrían contemplar la profundización del estudio incorporando nuevas variables en la clasificación de estructuras, tales como la toma de decisiones o la conflictividad en estos sistemas virtuales.

También podría resultar de interés la observación en relación a la aportación de estos grupos virtuales en la autopercepción de los individuos dentro y fuera de estas estructuras.

\section{NOTAS}

(1) Toledano, B. (2017). El número de usuarios que ha sumado Twitter en el último trimestre asciende a cero. El Mundo, 22 de julio

\section{Bibliografía}

Berrocal, S., Campos-Dominguez, E. y Redondo, M. (2014). Prosumidores mediáticos en la comunicación política: El «politainment en YouTube Comunicar, 43.

Caldevilla, D. (2009). Democracia 2.0: La política se introduce en las redes sociales. Pensar la Publicidad, 3 (2).

Cárdenas-Neira, C. (2014). Representación de la acción política de los estudiantes chilenos: Movilización de significados en Redes Sociales. Última Década, 22 (40).

Castells, M. (2008). Comunicación, poder y contrapoder en la sociedad red (II). Los nuevos espacios de la comunicación. Telos, 75.

Congosto, M.L. (2011). Twitter y política: información, opinión y ¿predicción? Cuadernos de Comunicación Evoca, 4

Dahl, R. (1961). Who governs? Yale University Press.

Deltell, L. (2011). Estrategias de comunicación política en las redes sociales durante la campaña electoral del 2011 en España: el caso de Equo. Actas de las Jornadas de la Asociación Madrileña de Sociología.

Ellison, N., Steinfield, C. y Lampe, C. (2007). The Benefits of Facebook Friends: Social Capital and College Students' Use of Online Social Network Sites. Journal of Computer Mediated Communication, 12(1). Doi:10.1111/ j.10836101.2007.00367.x.

Flórez, J. L. (2008) Análisis de redes sociales: el poder de la influencia. Harvard - Deusto Marketing \& Ventas, 86, $62-70$.

Grunig, J.E. y Hunt, T. (2003). Dirección de Relaciones Públicas. Barcelona: Ed Gestión 2000. 
Hunter, F. (1953). Community power structure: a study of decision makers. University of North Carolina Press.

IAB Spain (2017). Estudio anual de redes sociales 2016. Disponible en www.iabspain.net

Internet World Stats (2017). Internet User Statistics \& 2017 Population for the 53 European countries and regions. Disponible en ww w.internetworldstats.com.

Rey, C. (2017). ¿Sabes cuántos usuarios tiene WhatsApp en 2017? Androidphobia, 3 de febrero

Rodríguez Andrés, R. y Ureña Uceda, D. (2011). Diez razones para el uso de Twitter como herramienta en la comunicación política y electoral" Comunicación y Pluralismo, 10.

Tapia, A., Gómez, B., Herranz, JM. y Matellanes, M. (2010). Los estudiantes universitarios ante las redes sociales: cuestiones de uso y agrupación en estructuras elitistas o pluralistas. Vivat Academia, 113.

Tapia, A. (2015). Fundamentos de Relaciones Públicas. Madrid: Ed. Pirámide.

Tumasjan, A., Sprenger, T., Sandner, P. y Welpe, I. (2010). Predicting Elections with Twitter: What 140 Characters Reveal about Political Sentiment. Proceedings of the Fourth International AAAI Conference on Weblogs and Social Media. [Disponible en https://www.aaai.org/ocs/index.php/ICWSM/ICWSM10/paper/view/1441]

Túnez, M. y Sixto, J. (2011). Redes sociales, política y Compromiso 2.0: La comunicación de los diputados españoles en Facebook. Revista Latina de Comunicación Social, 66. Doi: 10.4185/RLCS-66-2011-930-210-234.

Walton, J. (1966). Discipline, method and community power: A note on the sociology of knowledge. American Sociologial Review, 31 .

Zarrella, D. (2010). New data: can Twitter predict elections? Blog de Dan Zarrella [Disponible en http:// danzarrella.com/new-data-can-twitter-predict-elections.html] 